\title{
Efectos del té de yerba mate, reflejado en el tiempo obtenido en el trabajo del día (WOD) en deportistas que practican CrossFit
}

\author{
Mate tea effects reflected in time performed in the work of the day \\ (WOD) in athletes who practice CrossFit
}

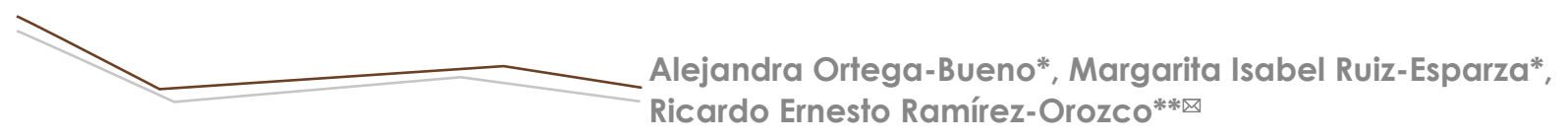

Ortega-Bueno, A., Ruiz-Esparza, M. I., \& Ramírez-Orozco, R. E. (2018). Efectos del té de yerba mate, reflejado en el tiempo obtenido en el trabajo del día (WOD) en deportistas que practican CrossFit. Investigación y Ciencia de la Universidad Autónoma de Aguascalientes, 26(73), 68-73.

\section{RESUMEN}

Actualmente el consumo de suplementos para mejorar el rendimiento ha aumentado sin considerar las consecuencias que ocasionan si no se toman apropiadamente. La yerba mate ha sido considerada una candidata contra enfermedades como la obesidad por sus múltiples efectos como: disminución en la diferenciación de adipocitos y triglicéridos, incremento de la tasa metabólica, función antioxidante y antiinflamatoria. Los beneficios en el deporte no han sido esclarecidos, por lo cual se consideró el estudio de los efectos del té de yerba mate en el rendimiento de deportistas que practican CrossFit habitualmente $(n=30)$. Treinta minutos antes de la prueba consumieron $300 \mathrm{ml}$ de té de yerba mate, el rendimiento fue evaluado en términos de tiempo total de ejecución de rutina. Se observó un efecto positivo en el tiempo de rutina

Palabras clave: té de yerba mate; rendimiento; CrossFit; ergogénico; suplemento; resistencia.

Keywords: mate tea; performance; Crossfit; ergogenic; supplement; resistance.

Recibido: 15 de marzo de 2017, aceptado: 13 de noviembre de 2017

* Centro de Ciencias de la Salud, Universidad Autónoma de Aguascalientes. Av. Universidad 940, Ciudad Universitaria, C. P. 20131, Aguascalientes, Aguascalientes, México. Correo electrónico: ale. ortegab@hotmail.com; isabelruizem@gmail.com

** Departamento de Nutrición y Cultura Física, Centro de Ciencias de la Salud, Universidad Autónoma de Aguascalientes. Av. Universidad 940, Ciudad Universitaria, C. P. 20131, Aguascalientes, Aguascalientes, México. Correo electrónico: licrero@hotmail.com

$\otimes$ Autor para correspondencia cuando el té de yerba mate fue ingerido (4:46 minutos vs. 4:12 minutos) $p=0.001$. Los aportes de este estudio resultan útiles para nuevas investigaciones en nutrición deportiva y rendimiento en atletas.

\section{ABSTRACT}

Nowadays, the use of nutritional supplements among athletes to improve performance has increased without considering the consequences that may result if not taken properly. However, the natural beverage mate tea is considered as candidate against diseases like obesity because of its multiple benefits, such as: a lower differentiation of adipocytes and triglycerides, an increase in the metabolic rate in addition to its antioxidant and anti-inflammatory function. Benefits in sport performance have not been elucidated yet, which led us to study yerba mate effects in sport performance of CrossFit athletes. Thirty athletes who usually practice CrossFit were evaluated. Mate tea $(300 \mathrm{ml}$ ) was consumed $30 \mathrm{mi}-$ nutes before the test; performance was evaluated in terms of the total time taken to complete a routine. A positive effect was noted on athletic performance when mate tea was consumed $(4: 46$ minutes vs. $4: 13$ minutes) $p=0,001$. The contributions of this study are useful for new research into sports nutrition and athlete performance.

INTRODUCCIÓN

En la actualidad existen componentes de alimentos naturales como el té de yerba mate (llex paragua- 


\section{InVESTIGACIÓn Y CIEnCIA DE LA UחIVERSIDAD AUTÓnOMH DE RGUASCALIEחTES}

riensis), que es consumido como una bebida de infusión, actualmente la yerba mate es considerada una de las más consumidas en el mundo, es distribuida en bolsas individuales como té o concentrado para suplemento en industrias de alimentos (Filip, López, Giberti, Coussio, \& Ferraro, 2001; Myung-Sook, Hyo Jin, Sang Ryong, Do Yeon, \& Un Ju, 2017). El té de yerba mate lleva un proceso de secado lento por medio del uso de humo de leña, denotando en la variedad de características reflejadas en el sabor, aroma y cambios en la composición química del té, de los cuales diversos componentes tales como polifenoles (ácido clorogénico), cafeína, flavonoides (quercetina, rutina y kaempferol) teobromina y alcaloides purínicos forman parte (Bastos, De Oliveira, Matsumoto, Carvalho, \& Ribeiro, 2007; Gómez-Juaristi, Martínez-López, Sarria, Bravo, \& Mateos, 2017; Heck \& de Mejía, 2007).

Algunos de los múltiples beneficios documentados en la literatura incluyen función antioxidante, mejora en la respuesta antiinflamatoria, además de una disminución en la diferenciación de adipocitos y triglicéridos; todo esto observado en diversos estudios animales (Gambero \& Ribeiro, 2015). Por otro lado, incluso se ha descrito una mejoría en modelos murinos de obesidad en el restablecimiento de niveles normales de glucosa, aminorando los efectos de la resistencia a la insulina (Kang et al., 2012).

La yerba mate es utilizada como droga vegetal o extractos en fármacos fitoterápicos para tratamientos contra el sobrepeso y la obesidad, además de como componente en suplementos dietéticos por su contenido de micronutrimentos y su acción energizante; sin embargo, sólo se ha reportado el efecto de aumentar la tasa metabólica basal y reducir el coeficiente respiratorio en periodos de descanso prolongados de 1-4 h) (Martinet, Hostettmann, \& Schutz, 1999).

Por otro lado, el Crossfit es un régimen de actividad física basado en la realización de movimientos funcionales en intervalos de alta intensidad, está constituido principalmente por componentes de estilo olímpico de levantamiento de pesas, pliometría y calistenia (Poston et al., 2016). Algunos de los ejercicios más comunes de esta disciplina son carrera a pie, gimnasia, ruedo y levantamiento de pesas; la combinación de frecuencia y alta intensidad entre estos implica poco tiempo de recuperación, lo que reporta una mejoría importante en la con- dición física (Ledon \& Tosti, 2017). Las sesiones de CrossFit normalmente están compuestas por cuatros partes: calentamiento, ejercicios de técnica y fuerza, el trabajo del día (WOD) y se finaliza con series de estiramientos (Smith, Sommer, Starkoff, \& Devor, 2015). En este contexto, la obtención de un mejor rendimiento conlleva a los deportistas a la búsqueda de alternativas, como lo es la suplementación nutricional (Sánchez-Oliver, Miranda León, \& Guerra Hernández, 2008).

Burke \& Deakin (2006) definen un suplemento nutricional como un producto que es ingerido por vía oral que contiene un ingrediente de la dieta para suplementar la alimentación o para mejorar el rendimiento con base en una marca impuesta ya sea por tiempo, velocidad, fuerza, coordinación, etc., por mencionar algunas características. Existe una variedad importante de suplementos, como vitaminas, minerales, proteínas, aminoácidos, preparaciones de medicina tradicional, extractos de hierbas, ácidos grasos esenciales, prebióticos, enzimas, entre otros compuestos y metabolitos (Burke \& Deakin, 2006).

El uso de suplementos en personas que realizan deporte ha ido en aumento de acuerdo con estudios que denotan que $48 \%$ aproximadamente de atletas universitarios consumen este tipo de ayudas ergogénicas (Woolsey, Waigandt, \& Beck, 2010). El deportista los ingiere con el fin de obtener un efecto ergogénico, es decir, una ayuda externa para mejorar el rendimiento físico de acuerdo con la disciplina llevada a cabo (figura 1).

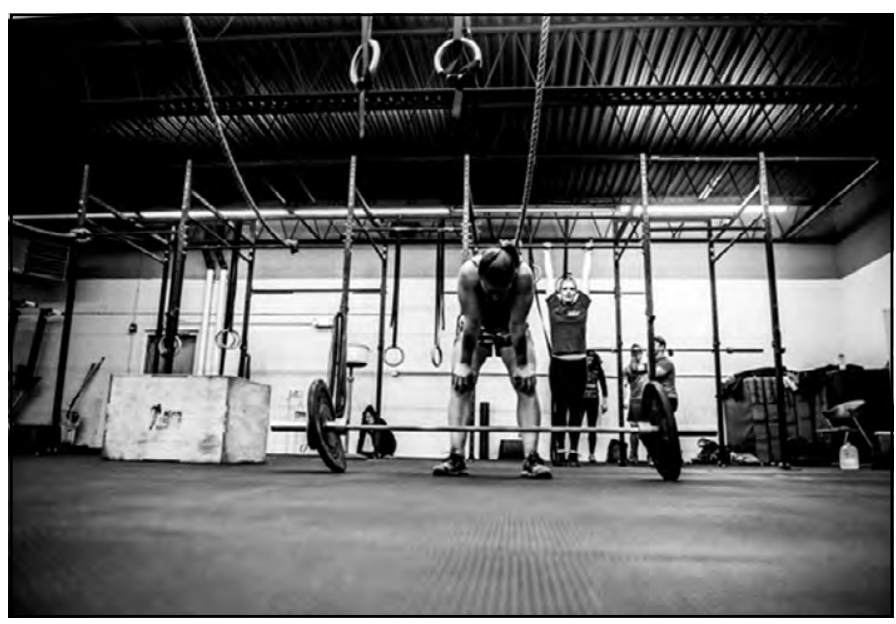

Figura 1. Una dieta deficiente y la falta de suplementación adecuada se ven reflejados en el rendimiento deportivo. Imagen de CrossfitBath (2017). 
Hoy día, la imagen corporal se ha vuelto una determinante en la sociedad, es una de las causas en el aumento de ayudas ergogénicas, lo que causa un abuso entre los asistentes a gimnasios y deportistas (Santesteban Moriones \& Ibáñez Santos, 2017). Debido a la problemática que existe por el abuso de suplementos se optó por buscar una mejor opción, orgánica y menos dañina que pueda aportar beneficios similares a los de las ayudas ergogénicas. La falta de evidencia de las propiedades del té de yerba mate con respecto a la mejoría en el desempeño deportivo permite plantear el objetivo de comprobar los efectos que causa el té de yerba mate en el rendimiento de deportistas reflejado en el tiempo de ejecución de la rutina del día en deportistas que practican CrossFit.

\section{MATERIALES Y MÉTODOS}

El estudio fue realizado en 30 deportistas que practican CrossFit en el gimnasio Infinity Fitness Center de la ciudad de Aguascalientes. Se tomaron en consideración los siguientes criterios: atletas que practican CrossFit con edades de 18 a 35 años de ambos sexos, sin limitación por alguna lesión, con un estado de salud óptimo, con y sin dominio de los ejercicios a realizar para la investigación. Se utilizaron remadoras Xebex® y pelotas medicinales GetRxd $\AA$ de $16 \mathrm{lb}$ para los hombres y de $14 \mathrm{lb}$ para las mujeres. EI WOD aplicado constó de tres ejercicios diferentes realizados a un máximo esfuerzo.

\section{Ejercicios del WOD}

Remo en un tramo de 250 metros: ejercicio que consiste en colocarse en la máquina de remo, sentarse en la misma, y posicionar los pies en los pedales, se toma el manillar con los brazos extendidos realizando el gesto de remo hasta llegar hacia el pecho y después devolver el manillar hacia enfrente haciendo una flexión de piernas.

- 20 Burpees: consiste en bajar a sentadilla hasta tocar el suelo con las manos y sin despegarlas del mismo para después impulsar ambas piernas a la vez hacia atrás, hasta quedar en posición para realizar una lagartija y una vez realizado, se regresan ambas piernas acercando las rodillas al pecho para subir por completo y finalizar con un salto subiendo las manos y dando un aplauso por encima de la cabeza.

- 20 disparos de pelota: consisten en tomar una pelota medicinal con ambas manos frente al pecho, bajar en sentadilla rompiendo

ligeramente el ángulo de $90^{\circ}$ para después subir e inmediatamente lanzar la pelota hacia arriba tocando la pared a una altura de 9 pies $(2.74$ m) y finalmente interceptar la pelota y repetir el ejercicio de manera continua.

\section{Suplementación de té de yerba mate}

Se administró una toma de $300 \mathrm{ml}$ de agua con un contenido de $30 \mathrm{~g}$ de yerba mate Rosamonte ${ }^{\circledR}$ dejando reposar el té por 15 min con agua purificada a $70{ }^{\circ} \mathrm{C}$. Este fue tomando 30 minutos antes de realizar la prueba.

\section{Dieta, descanso y realización del WOD}

Se consideraron factores como la dieta, horas de descanso y rutinas anteriores, por lo que con tres semanas de anticipación se estructuró un plan de alimentación de acuerdo con sus necesidades de actividad física de tipo isocalórico que no modificara su peso, el plan fue estructurado de acuerdo a menús establecidos y estandarizados para disminuir la presencia de sesgos; además de continuar con la misma rutina se asesoró a los participantes sobre la ingesta diaria de alimentos en el mismo horario, así como las horas de descanso y sueño. Con respecto a la realización del WOD, todo fue cronometrado y verificado por el responsable y entrenador asegurando la correcta realización del ejercicio. Durante la primera sesión, se realizó de manera rutinaria el WOD sin tomar el té, después de una semana se volvió a realizar la sesión en esta ocasión con la toma del té. Las pruebas se realizaron en el mismo horario, esto se hizo por triplicado para validar la confiabilidad de los datos recabados.

\section{Análisis estadístico}

Los datos fueron representados como: media, desviación estándar, rango y porcentajes. Se verificó la distribución de variables mediante la prueba de Shapiro-Wilk, se utilizaron pruebas como: $t$ de Student para muestras independientes para observar diferencias entre género y $t$ de Student Pareada, se consideró un nivel de significancia de $p<0.05$. El análisis de los datos se llevó a cabo mediante el programa SPSS versión 20.

RESULTADOS

Se estudió a 30 deportistas que practican Crossfit del gimnasio Infinity Fitness Center de la ciudad de Aguascalientes, México; con un rango de edad de 18 a 35 años, la media de edad de la población estudiada fue de $26.23 \pm 5.0$ años, cabe mencionar 
IIVESTIGACIÓn Y CIEnCIR DE

LA UחIVERSIDAD AUTÓNOTH

DE RGUASCALIETTES

Tabla 1

Tiempo cronometrado: mínimo y máximo

\begin{tabular}{cccc}
\hline Variable & Media $\pm \mathrm{DE}$ & Mínimo & Máximo \\
\hline Sin té de yerba mate (min:seg) & $4: 46 \pm 0.86$ & $2: 58$ & $6: 10$ \\
Con té de yerba mate (min:seg) & $4: 12 \pm 0.78$ & $3: 06$ & $6: 09$ \\
\hline
\end{tabular}

Nota: Datos representados en rangos, media y desviación estándar. $n=30$.

Elaboración propia.

Tabla 2

Suplementación de té de yerba mate y tiempo para completar entrenamiento $(n=30)$

\begin{tabular}{|c|c|c|c|}
\hline & $\begin{array}{l}\text { Sin ingestión de té de yerba } \\
\text { mate (min:seg) }\end{array}$ & $\begin{array}{l}\text { Con ingestión de té de yerba } \\
\text { mate (min:seg) }\end{array}$ & $\mathrm{p}$ \\
\hline Género masculino & $4: 41 \pm 1.0$ & $4: 01 \pm 0.80$ & 0.015 \\
\hline Género femenino & $4: 49 \pm 0.72$ & $4: 24 \pm 0.78$ & 0.035 \\
\hline Población total & $4: 46 \pm 0.86$ & $4: 12 \pm 0.78$ & 0.001 \\
\hline
\end{tabular}

que $50 \%$ de la muestra representa cada género (15 hombres y 15 mujeres). Los datos antropométricos fueron los siguientes: peso: $71.6 \pm 11.1 \mathrm{~kg}$, talla: $1.69 \pm$ $0.06 \mathrm{~m}$, IMC: $24.8 \pm 3.6 \mathrm{~m} / \mathrm{kg}^{2}$, cabe mencionar que de acuerdo con su índice de masa corporal fueron clasificados de la siguiente manera: normopeso: $56.7 \%(n=17)$, sobrepeso: $36.7 \%(n=11)$ y obesidad: $(n=2)$. Los resultados con base en el rendimiento por tiempo cronometrado indicaron una mejora en la eficiencia para realizar el WOD con una media de 32.7 segundos menos en comparación con la primer sesión realizada (4:46 vs. 4:13 minutos) $p=0.001$ (tabla 1).

En cuanto al rendimiento deportivo con base en el tiempo cronometrado de acuerdo al género después de consumir el té de yerba mate se encontró que las mujeres obtuvieron menor tiempo en comparación con el género masculino de 23 segundos de diferencia (4:24 vs. 4:01 min) $p=0.385$; no obstante, no representa diferencias estadísticas entre géneros; sin embargo, cabe mencionar que esta mejora en el tiempo de ejecución de rutina es notoria al momento de estratificar por género, como muestran la tabla 2 y la figura 2.

\section{DISCUSIÓN}

Actualmente existe una gran variedad de suplementos deportivos, entre ellos las bebidas ergogénicas, de las cuales muchas son utilizadas de manera excesiva sin medir las consecuencias negativas que

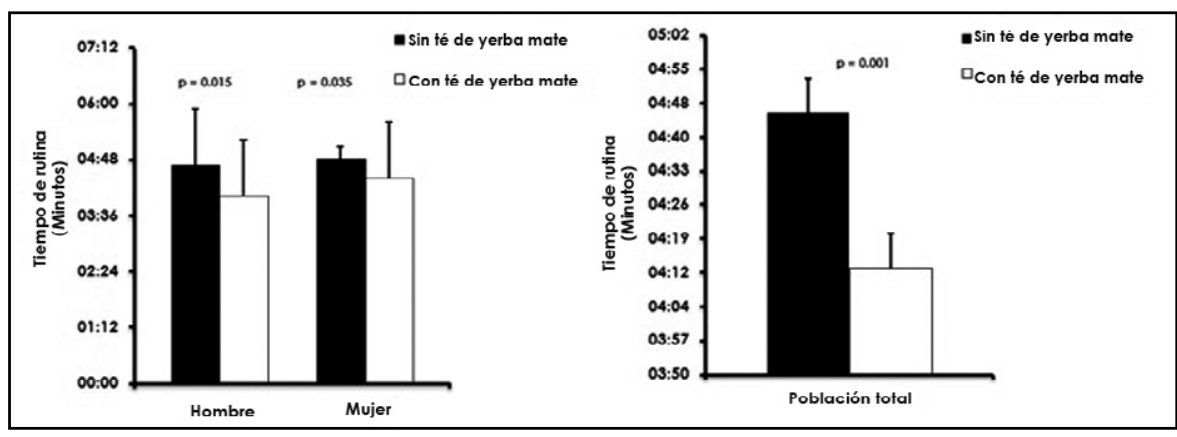

Figura 2. Tiempo cronometrado de rutina de acuerdo con género y población total. (lzquierda) Medición del rendimiento deportivo con base en el tiempo antes y después de la suplementación con té de yerba mate por género $(n=15)$. (Derecha) Medición del rendimiento deportivo con base en el tiempo antes y después de la suplementación con té de yerba mate en población total $(n=30)$.

Elaboración propia. 
IIVESTIGACIÓn Y CIERCIA DE LA UNIVERSIDAD AUTÓNOMA DE AGUASCALIERTES pueden ocasionar en la salud, ya que no se conocen por completo sus componentes o el proceso por el que fueron sometidas (Sánchez Oliver et al., 2008). Se buscó comprobar las propiedades de este té como bebida ergogénica. Estudios de Schubert, Astorino, \& Leal Azevedo (2013) apoyan la idea de considerar el té de la sustancia estudiada como parte de una suplementación nutricional por su acción energizante debido a la cafeína natural que contiene, lo cual ha incrementado el consumo de productos que contienen yerba mate (Schubert et al., 2013).

La yerba en estudio es una planta rica en compuestos orgánicos y vitaminas, sus propiedades antioxidantes y energizantes han sugerido una mejora en la optimización de la absorción nutricional de esta manera mejorando el rendimiento de los deportistas (Maiocchi et al., 2016); al consumir esta bebida, los resultados de este trabajo reflejan un efecto positivo en el rendimiento en términos de menor tiempo en la realización del WOD. Ello pudiera deberse al efecto agudo de la cafeína contenida en la yerba mate, la medición en situación de ayuno puede esclarecer mejor el efecto inmediato en el ejercicio evitando sesgos de factores como la dieta, descanso, entre otros; sin embargo, debido al tipo de disciplina, esto puede comprometer al deportista debido a la alta demanda energética requerida en este tipo de actividad física, lo que afecta su salud.

Alkhatib describe el efecto en el aumento de la tasa oxidativa de grasas durante la actividad física y, por ende, la energía proveniente de ácidos grasos, lo que puede ser una explicación en la mejora del rendimiento, esto se ha propuesto como parte del tratamiento de las estrategias focalizadas a la pérdida de peso (Alkhatib, 2014). Por otro lado, Schubert et al. (2013) estudiaron el efecto en el rendimiento al administrar tres bebidas "energizantes" (Placebo, Guayakí Yerba Maté Organic Energy Shot TM, Red Bull Energy Shot TM) en atletas corredores, y no encontraron diferencias en el rendimiento en los que tomaban estas bebidas (Schubert et al., 2013), a diferencia de nuestros resultados hubo una disminución en el tiempo de la realización del WOD de los deportistas de Crossfit, lo que puede deberse al efecto agudo de la cafeína y del aumento en la tasa metabólica anteriormente mencionado, al optimizar el uso de energía durante la actividad física; sin embargo, es necesario comprobar este efecto en otro tipo de deportes de corta y larga duración y evaluar de mejor manera este beneficio.
Al analizar la problemática que existe sobre el consumo excesivo de suplementos en deportistas y los problemas de dopaje, conocer a fondo los efectos de la yerba mate y de sus componentes puede ser considerado una opción viable de bebida para deportista sin sanción por dopaje. Alfredo Cabañes \& Salinero (2013) estudian el consumo de cafeína durante el entrenamiento y competición basados en la normativa antidopaje; en ello se ha observado un efecto positivo reflejado en fuerza y resistencia en el deportista sin miedo a resultar positivo en la prueba de dopaje (Alfredo Cabañes \& Salinero, 2013). Hoy en día, a causa de los beneficios aportados por esta sustancia, 3 de cada 4 deportistas consumen cafeína antes o durante una competición; esto ha sido observado principalmente en deportes de resistencia (Del Coso, Muñoz, \& Muñoz-Guerra, 2011).

La realización de futuras investigaciones con un mayor control en variables que puedan sesgar los resultados en el desempeño de los atletas, como consumo de medicamentos, tabaco y alcohol, ayudas ergogénicas, horas de sueño, puede contribuir a un mejor entendimiento sobre los efectos reales de la yerba mate, además de poner a prueba otros posibles beneficios potenciales, entre ellos la disminución de lípidos en sangre por ejemplo. La realización de este primer estudio piloto nos da pauta para lograr un mejor control sobre aspectos como interacción de nutrimentos, suplementación de manera individual o conjunta entre otros, lo cual puede permitir en un futuro el desarrollo de estrategias que permitan mejorar el rendimiento en el deportista.

CONCLUSIONES

Tanto en la disciplina de CrossFit como en cualquier otro deporte, es importante el desempeño y mejora constante en los principales componentes del rendimiento deportivo como velocidad, fuerza y coordinación, ya que permitirá tener un mejor posicionamiento y/o resultado en el entrenamiento y competencias. Nuestros datos sugieren el continuo estudio de la ingestión de té de yerba mate antes del ejercicio que permitirá esclarecer los beneficios y efectos secundarios; sin embargo, con base en los resultados positivos obtenidos, el té de yerba mate puede llegar a ser considerado una opción de suplementación nutricional una vez elucidados a fondo sus efectos en el deportista, la interacción con la dieta, descanso y el tipo de actividad física. 


\section{IIVESTIGAGIÓn Y CUERCIA DE LA UחIVERSIDAD AUTÓNOMA DE AGUASCALIERTES}

\section{Perspectivas del estudio}

Al ser un primer estudio piloto, se ha considerado para estudios posteriores variables como pruebas de esfuerzo, variabilidad de la frecuencia cardiaca, pruebas que permitan la medición de resistencia, fuerza, potencia y velocidad; de manera individual y conjunta, esto permitirá elucidar de una manera más adecuada los beneficios aportados por la yerba mate como parte de la suplementación deportiva.

\section{REFERENCIAS}

- Alfredo Cabañes, J. J., \& Salinero, J. C. (2013). La ingestión de una bebida energética con cafeína mejora la fuerza-resistencia y el rendimiento en escalada deportiva. Archivos de Medicina del Deporte, 30(4), 215-220.

- Alkhatib, A. (2014). Yerba Maté (Illex Paraguariensis) ingestion augments fat oxidation and energy expenditure during exercise at various submaximal intensities. Nutrition \& Metabolism, $11,42$.

- Bastos, D. H. M., De Oliveira, D. M., Matsumoto, R. L. T., Carvalho, P. O., \& Ribeiro, M. L. (2007). Yerba maté: Pharmacological properties, research and biotechnology. Medicinal and Aromatic Plant Science and Biotechnology, 1 (1), 37-46.

- Burke, L., \& Deakin, V. (2006). Clinical Sports Nutrition (3rd. ed., pp. 485-580). Sydney-NY: McGraw-Hill.

- Crossfitbath. (2017). Blog: WOD19-01-2018 [Fotografía]. Recuperado de http://crossfitbath.com/wod-19-118/\#iLightbox[gallery10604]/0

- Del Coso, J., Muñoz, G., \& Muñoz-Guerra, J. (2011). Prevalence of caffeine use in elite athletes following its removal from the World Anti-Doping Agency list of banned substances. Applied Physiology Nutrition, and Metabolism, 36 (4), 555-561.

- Filip, R., López, P., Giberti, G., Coussio, J., \& Ferraro, G. (2001). Phenolic compounds in seven South American Ilex species. Fitoterapia, 72 (7), 774-778.

- Gambero, A., \& Ribeiro, M. L. (2015). The positive effects of yerba maté (Ilex paraguariensis) in obesity. Nutrients, 7(2), 730750.

- Gómez-Juaristi, M., Martínez-López, S., Sarria, B., Bravo, L., \& Mateos, R. (2017). Absorption and metabolism of yerba mate phenolic compounds in humans. Food Chemistry, 240, 10281038. doi: 10.1016/j.foodchem.2017.08.003

- González-Gallego, J., Garrido Pastor, J., Mataix Verdú, J., \& Villegas García, J. (1988). Nutrición y ayudas ergogénicas en el deporte. Arch. Medicina del Deporte.

- Heck, C. I., \& De Mejia, E. G. (2007). Yerba Mate Tea (Ilex paraguariensis): A comprehensive review on chemistry, health implications, and technological considerations. Journal of Food Science, 72(9), R138-R151.

- Kang, Y. R., Lee, H. Y., Kim, J. H., Moon, D. I., Seo, M. Y., Park, S. H.,...Oh, H. G. (2012). Anti-obesity and anti-diabetic effects of
Yerba Mate (Ilex paraguariensis) in C57BL/6J mice fed a highfat diet. Laboratory Animal Research, 28(1), 23-29.

- Ledon, J. A., \& Tosti, A. (2017). CrossFit-associated allergic contact dermatitis. Dermatitis, 28(6), 368. doi: 10.1097/ DER.0000000000000321

- Maiocchi, M. G., Del Vitto, L. A., Petenatti, M. E., Marchevsky, E. J., Avanza, M. V., Pellerano, R. G., \& Petenatti, E. M. (2016). Composición multielemental y valor nutricional de "dumosa" (llex dumosa), "yerba mate" (I. paraguariensis) y su mezcla comercial en diferentes formas de uso. FCA UNCUYO, 48(1), 145-159.

- Martinet, A., Hostettmann, K., \& Schutz, Y. (1999). Thermogenic effects of commercially available plant preparations aimed at treating human obesity. Phytomedicine, 6(4), 231-238.

- Myung-Sook, C., Hyo Jin , P., Sang Ryong, K., Do Yeon, K., \& Un Ju , J. (2017). Long-term dietary supplementation with yerba mate ameliorates obesity and metabolic disorders in mice by regulating energy expenditure and lipid metabolism. Journal of Medicinal Food, 20(12), 1168-1175.

- Poston, W. S., Haddock, C. K., Heinrich, K. M., Jahnke, S. A., Jitnarin, N., \& Batchelor, D. B. (2016). Is high-intensity functional training (HIFT)/crossfit safe for military fitness training? Mil Med, $181(7), 627-637$.

- Sánchez Oliver, A. J., Miranda León, M. T., \& Guerra Hernández, E. (septiembre de 2008). Estudio estadístico del consumo de suplementos nutricionales y dietéticos en gimnasios. ALAN Archivos Latinoamericanos de Nutrición, 58(3), 221-227.

- Santesteban Moriones, V., \& Ibáñez Santos, J. (2017). Ayudas ergogénicas en el deporte. Nutrición Hospitalaria, 34 (1), 204-215.

- Schubert, M. M., Astorino, T. A., \& Leal Azevedo, J. (2013). The effects of caffeinaited "energy shots" on time trial performance. Nutrients, 5(6), 2062-2075.

- Smith, M. M., Sommer, A. J., Starkoff, B. E., \& Devor, S. T. (2015). Crossfit-based high-intensity power training improves maximal aerobic fitness and body composition. Journal of Strength and Conditioning Research, 29(10): el. doi: 10.1519/ JSC.0b013e318289e59f

- Woolsey, C., Waigandt, A., \& Beck, N. C. (2010). Athletes and energy drinks: Reported risk-taking and consequences from the combined use of alcohol and energy drinks. Journal of Applied Sport Psychology, 22(1), 65-71. 